\title{
Coupled dipole plasmonics of nanoantennas in discontinuous, complex dielectric environments
}

\author{
Gregory T. Forcherio, ${ }^{a}$ Phillip Blake, ${ }^{b}$ Manoj Seeram, ${ }^{b}$ Drew DeJarnette, ${ }^{c}$ D. Keith \\ Roper $^{\mathrm{a}, \mathrm{b}, \dagger}$ \\ ${ }^{a}$ MicroElectronics-Photonics Program, University of Arkansas, Fayetteville, Arkansas 72701, USA \\ ${ }^{\mathrm{b}}$ Ralph E. Martin Department of Chemical Engineering, University of Arkansas, Fayetteville, Arkansas \\ 72701, USA \\ 'Department of Mechanical Engineering, University of Tulsa, Tulsa, OK 74104, USA \\ †Corresponding author. E-mail: dkroper@uark.edu, phone: (479) 575-6691, fax: (479) 575-7926.
}

\section{Revised for Journal of Quantitative Spectroscopy \& Radiative Transfer.}

Two-dimensional metamaterials support both plasmonic and coupled lattice (Fano) resonant modes that together could enhance optoelectronics. Descriptions for plasmon excitation in Fano resonant lattices in non-vacuum environments typically use idealized, homogeneous matrices due to computational expense and limitations of common approaches. This work described both localized and coupled resonance activity of two-dimensional, square lattices of gold ( $\mathrm{Au})$ nanospheres (NS) in discontinuous, complex dielectric media using compact synthesis of discrete and coupled dipole approximations. This multi-scale approach supported attribution of experimentally observed spectral resonance energy and bandwidth to interactions between metal and dielectric substrate(s) supporting the lattices. Effective polarizabilities of single AuNS, either in vacuo or supported by glass and/or indium tin oxide (ITO) substrates, were obtained with discrete dipole approximation (DDA). This showed plasmon energy transport varied with type of substrate: glass increased scattering, while ITO increased absorption and energy confinement. Farfield lattice interactions between AuNS with/without substrates were computed by coupled dipole approximation (CDA) using effective polarizabilities. This showed glass enhanced diffractive features (e.g., coupled lattice resonance), while ITO supported plasmon modes. This compact, multiscale approach to describe metasurfaces in complex environments could accelerate their development and application.

Keywords: plasmonics, metamaterials, nanoantenna, nanoparticle, dipole approximation, Fano resonance 


\section{Introduction}

Charge polarization of nanoantennas depends on local dielectric environment [1], as described by Clausius-Mossotti [2,3] for electrostatic dipoles embedded in a homogeneous reference. A compact description of localized and coupled resonant modes due to polarization of ordered nanoantennas in complex, discontinuous dielectric environments is needed for energy harvesting [4,5], sensors [6,7], photonic circuits [8,9], and nonlinear optics [10,11]. This calls for a multiscale, electrodynamic approach beyond dielectric averaging, analytic approximations, or present computational methods. Mass-averaged dielectric values have been shown to oversimplify plasmon and lattice resonant modes [12]. Spectrum-averaged refractive indices insufficiently describe nanoantennas supported by emergent materials like transition metal dichalcogenides [13]. Maxwell-Garnett and effective medium [14-17] analytic approaches describe mode energy shifts for random [17-19] and ordered [20-22] nanospheres using Mie theory and the coupled dipole approximation (CDA), respectively, but are limited to real-valued dielectric substrates with nearzero dispersion.

Finite difference time domain (FDTD), boundary/finite element methods (BEM/FEM), and discrete dipole approximation (DDA) are used to describe polarizable electric resonance modes for arbitrarily-shaped nanostructures embedded in a non-dispersive, uniform dielectric environment [23-25] or between dielectric super- and sub-strates [25-31]. Discretization of time and space in FDTD and FEM constrain efficient computation of locally complex dielectrics, particularly for large-area metasurfaces. BEM reduces memory usage, but is limited to describing nanoantennas whose volume may be approximated as a surface [32], e.g., nanodiscs. DDA has been used to compute frequency dependent polarization for single nanoantenna on complex dielectrics at less computational expense than FDTD by discretizing structures into cubic lattices of point dipoles with unique dielectric behavior [33,34]. Schatz et al. used DDA to predict experimental localized surface plasmon resonances (LSPR) from single nanospheres and nanotetrahedrons supported by glass and mica substrates [27,35]. However, coupled plasmon modes between nanoantenna in lattice arrangements described by DDA to date have omitted rigorous analysis of substrate to ease computational expense [36,37]. Comprehensive description of nanoantennas in two-dimensional metamaterials supported by dispersive, complex dielectric substrates requires a more complete approach.

This work inserts effective single-particle polarizabilities computed by DDA in complex (both real and lossy) dielectrics into a rapid, semi-analytical coupled dipole approximation (rsaCDA) to efficiently describe resonant plasmon, diffractive, and coupled lattice modes for large area, regular lattices of nanoantennas. Predominant features observed in transmission UV-vis spectra from a square lattice of $80 \mathrm{~nm}$ radius gold nanospheres (AuNS) supported by indium tin oxide (ITO)-covered glass were reproduced by integrating DDA results at $100 \mathrm{~nm}$ with rsa-CDA calculations at $>100,000 \mathrm{~nm}$ scales. This multiscale DDA/rsa-CDA approach showed that glass substrates increased resonant scattering which contributed to diffractive features (e.g., coupled lattice resonance) in array spectra; while lossy ITO substrates increased absorption and enhanced energy confinement, contributing to dipole plasmon features in the spectra. 


\section{MATERIALS AND METHODS}

\subsection{Numerical Modeling}

The CDA describes electrodynamics of a nanoantenna lattice comprising a metasurface by treating each constituent antenna as a point dipole with scalar polarizability [20,38,39]. Charge density polarization of each structure, $\boldsymbol{P}$, is calculated according to antenna polarizability $(\alpha)$ and cumulative contributions from the incident field, $\boldsymbol{E}_{o}$, and adjacent dipoles comprising the lattice, $\boldsymbol{E}_{\text {lat }}$, viz.,

$$
\boldsymbol{P}=\alpha\left(\boldsymbol{E}_{o}+\boldsymbol{E}_{\text {lat }}\right)
$$

The retarded dipole sum calculates $\boldsymbol{E}_{\text {lat }}$ by summing interactions of all nanoantennas in the lattice on each individual antenna. An analytical $\alpha$ is available for symmetric shapes such as spheres, spheroids, and toroids [35,40,41] in homogenous media, but external numerical calculation (e.g., DDA) is required for complex shapes [42] or most cases of non-uniform media. Polarizability corrections exist for spherical nanoantenna within non-lossy, multi-layered media [43], however they are not extendable to arbitrary antenna shapes or lossy substrates.

This work used DDA package DDSCAT 7.3 to compute effective polarizabilities of $80 \mathrm{~nm}$ radius AuNS in a vacuum and mounted on ITO or soda lime glass substrates [44-46]. DDSCAT solves Maxwell's equations by discretizing arbitrary geometries into a cubic lattice of point dipoles. Targets were discretized according to their Cartesian description using a MATLAB (v8.3, MathWorks, Natick, MA, USA) tool available on nanoHUB [47]. Each point dipole within a target was assigned a polarizability, $\alpha_{d i}$, according to the "lattice dispersion relation" described by Gutkowicz-Krusin [48] to permit calculation of its polarization $\boldsymbol{P}_{d i}$ in response to $\boldsymbol{E}_{o}$. DDSCAT was modified to output complex effective polarizability, $\alpha_{e f f}$, for each AuNS-substrate pair by summing the polarizability of each dipole within the target [7,42], calculated as a function of the inter-dipole spacing $\left(d_{d i}\right), \boldsymbol{P}_{d i}$ for the $i$ th dipole per unit volume, and $\boldsymbol{E}_{o}$ at the $i$ th dipole:

$$
\alpha_{e f f}=\sum_{i=1}^{n} \frac{d_{d i}{ }^{3}\left(\boldsymbol{P}_{d i}\right)_{i}}{\left(\boldsymbol{E}_{o}\right)_{i}}
$$

DDA simulations were performed with $d_{d i}=5 \mathrm{~nm}$ for incident vacuum wavelengths between $400 \mathrm{~nm}$ and $800 \mathrm{~nm}$ at $1 \mathrm{~nm}$ resolution on a 16-core supercomputer node with $32 \mathrm{~GB}$ of memory. Near-field enhancement plots were generated using $d_{d i}=2 \mathrm{~nm}$ for convergence. Wave vector $(k)$ was incident orthogonal to the substrate (along $\mathrm{x}$-axis) and polarized along the $\mathrm{z}$-axis. Substrates were modeled as a cylinder in the $y-z$ plane with radius and height equal to $2 x$ the particle radius [27,35] i.e., $160 \mathrm{~nm}$. Interaction between AuNS and substrate was considered at $0 \%, 15 \%$, and $30 \%$ particle surface area for two scenarios: (1) AuNS embedded into the substrate and (2) AuNS "cut-off" at the substrate surface. Percentage interaction values were based on analytic weighting factors characteristic of 1/e plasmon interaction distance from the nanoantenna surface [17]. Dielectric data for soda lime glass and ITO reported by Rubin and König et al., respectively, were used $[49,50]$. 
Computed polarizabilities were then used to calculate far-field lattice spectra from square $301 \times 301$ arrays of AuNS using rsa-CDA [7,42]. The rsa-CDA [20,38,40] reduced computational time by taking advantage of $\pi / 2$ rotational symmetry in square lattices [40]. Dielectric data for $\mathrm{Au}$ reported by Johnson and Christy was used in all simulations [51]. This multi-scale DDA/rsa-CDA technique has been shown to reduce computation time more than 40,000-fold for nanoring lattices in vacuo relative to full volume DDA models [42].

\subsection{Sample Fabrication and Characterization}

A square lattice of AuNS with radii of $80 \mathrm{~nm}$ was lithographed using electron-beam lithography (EBL) and metallized by electroless plating [21,52]. Briefly, ITO-covered glass (300 nm ITO thickness) was sensitized with $\mathrm{SnCl}_{2}$. This substrate was electron resist spin-coated with poly(methylmethacrylate) (PMMA). EBL was performed with a Nanometer Pattern Generation System (JC Nabity Lithography Systems, Bozeman, MT) integrated on a scanning electron microscope (SEM) (Philips XL30, FEI, Hillsboro, OR). A 1:3 (volume) solution of methyl isobutyl ketone (MIBK) to isopropanol (IPA) was used for resist development. Electroless $\mathrm{Au}$ plating was performed by $\mathrm{AgNO}_{3}$ immersion for 2 minutes followed by $\mathrm{Na}_{3}\left[\mathrm{Au}\left(\mathrm{SO}_{3}\right)_{2}\right]$ immersion to galvanically displace $\mathrm{Ag}$ with $\mathrm{Au}$. Resist was removed with hot acetone. Deposited $\mathrm{Au}$ cylinders were flash annealed at $800{ }^{\circ} \mathrm{C}$ for three 90 second intervals to produce a square lattice of AuNS.

Figure 1 shows (a) an SEM micrograph of representative spheroids in the lattice; (b) a cross-section schematic; and (c) transmission UV-vis extinction spectra. An average sphere diameter of $161 \pm 6 \mathrm{~nm}$ and $630 \pm 18 \mathrm{~nm}$ lattice constant were extracted from SEM micrographs with a particle count of 226 [21]. Spectral characterization using a custom transmission UV-vis spectroscopy system [53] revealed coupled plasmon and lattice resonances at $528 \mathrm{~nm}$ and $665 \mathrm{~nm}$, respectively, with air above the AuNS on ITO-coated glass (solid line). These resonances shifted to $544 \mathrm{~nm}$ and $685 \mathrm{~nm}$, respectively, upon immersion into water (dashed line). 


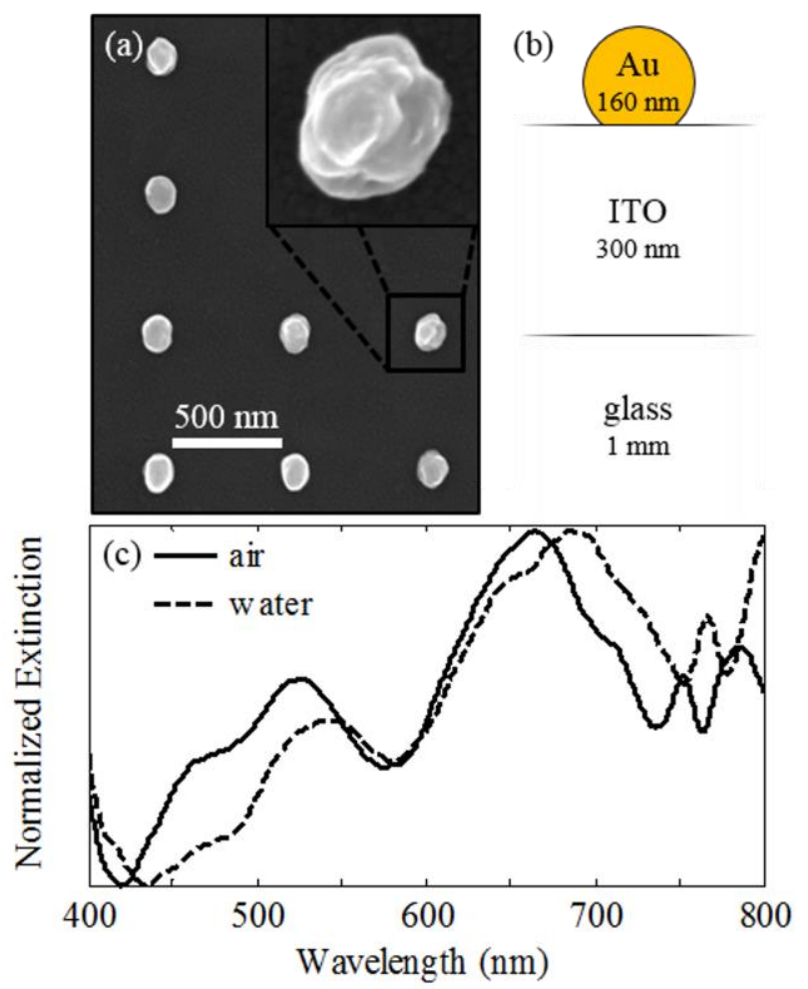

Figure 1. (a) SEM image, (b) cross-sectional material schematic, and (c) experimental extinction spectra of electroless-plated gold nanosphere lattice in air (solid) and water (dashed) environments.

\section{Results \& Discussion}

\subsection{Substrate effects on an isolated sphere: LSPR and near field interactions}

Discrete dipole approximation (DDA) simulation of electrodynamic polarizability for subwavelength, noble metal nanoantennas approached analytic Mie theory. Real and imaginary components of polarizability relate directly to near- and far-field optical behavior of subwavelength structures, respectively. Figure 2 shows real (blue lines) and imaginary (red lines) components of polarizability $(\alpha)$ for an $80 \mathrm{~nm}$ radius gold nanosphere (AuNS) in a vacuum calculated by Mie (solid) and DDA (dashed). Polarizability maxima calculated at $708 \mathrm{~nm}$ and 570 $\mathrm{nm}$ for $\operatorname{Re}(\alpha)$ and $\operatorname{Im}(\alpha)$ components, respectively, were within $2 \%$ of the analytic Mie solution. Differences in polarizability magnitude and dispersion in Figure 2 were attributable to geometry discretization in the DDA. The $\operatorname{Im}(\alpha)$, proportional to the extinction cross section, calculated by DDA in Figure 2 produced a $561 \mathrm{~nm}$ dipole localized surface plasmon resonance (LSPR) in the corresponding extinction efficiency spectra (see Figure 3). This was within $1 \%$ of the wavelength calculated using Mie theory. Accuracy of these DDA results support its description of polarizability, electromagnetic fields, and spectra for arbitrarily-shaped single nanoantennas, e.g., nanorings [54], in complex dielectric environments. 


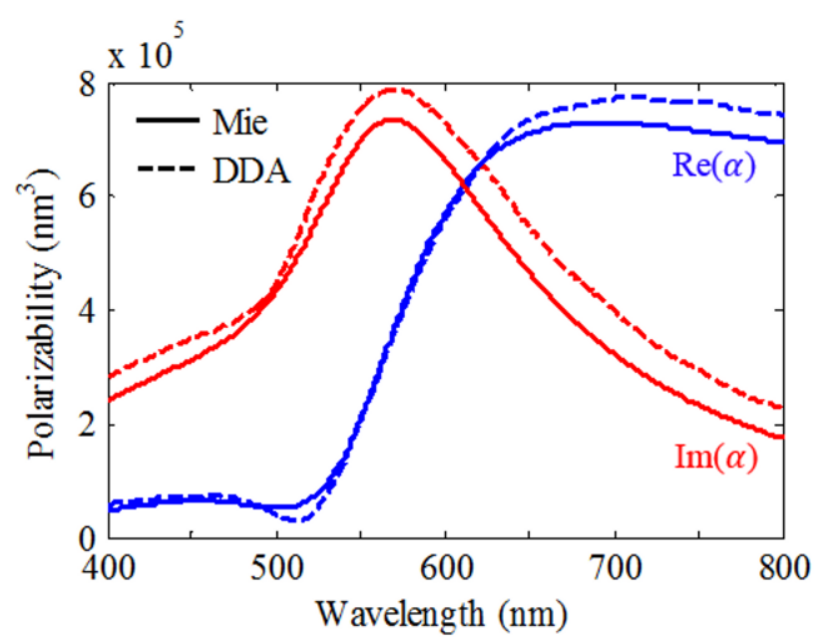

Figure 2. Real (blue) and imaginary (red) parts of dipole polarizability for an $80 \mathrm{~nm}$ radius Au nanosphere in a vacuum calculated by the DDA (dashed) and Mie theory (solid).

Plasmon modes and decay routes of AuNS fabricated on glass or indium tin oxide (ITO) were altered relative to Mie simulations in a vacuum. Figure 3(a) compares spectra from an 80 $\mathrm{nm}$ radius AuNS in vacuo (black) vs. supported on (solid lines) glass (blue) and ITO (red) substrates. The LSPR red-shifted $14 \mathrm{~nm}$ and $49 \mathrm{~nm}$ on glass and ITO, respectively, as atomic polarization in the substrate counteracted the Coulombic restoring force and decreased energy required to excite plasmons on the AuNS [22]. Previously, LSPR red-shifts from nanoantennas at an interface between two dielectrics (e.g., air above glass) have been estimated with an effective RI environment using Maxwell-Garnett [14] or weighted-average [17] dielectric approximations or polarizability corrections [43]. Validity of these approaches is limited to real-valued dielectrics with near-zero dispersion; neither has been used to describe non-spherical nanoantennas in lossy dielectrics. Conversely, arbitrary structures and complex dielectric environments are readily described using DDA.

DDA results in Figure 3(a) further show that embedding AuNS to 15\% (dashed) and 30\% (dotted) of its surface area into an oxide, either glass or ITO, decreased plasmon excitation energy and intensity. In glass, embedding the AuNS redshifted the LSPR $20 \mathrm{~nm}$ and $55 \mathrm{~nm}$ at $15 \%$ and $30 \%$, respectively, relative to $0 \%$ embedment. In ITO, the relative redshift was $44 \mathrm{~nm}$ and $95 \mathrm{~nm}$, respectively. ITO thickness on the fabricated sample was sufficiently thick (300 nm, see Figure 1) to neglect glass in the ITO simulations. Plasmon energy and intensity of silver nanorods have been shown to decrease as a result of substrate interaction using FDTD [55].

Figure 3(b) shows that compared to in vacuo conditions (black circle), introducing a glass (blue squares) or ITO (red squares) substrate increased resonant scattering relative to absorption depending on the percentage of AuNS embedment, $0 \% 15 \%$ and $30 \%$ surface area, and type of substrate. Use of the DDA elucidated increased scattering decay routes relative to vacuum. Radiative loss modes [22] accrued by substrates could include plasmon re-radiation, Hertzian scattering, or Raman scattering. Increased scattering decay routes is consistent with augmented LSPR bandwidths [56] in Figure 3(a). For glass, a real-valued dielectric, the ratio of scattering to absorption remained nearly constant as embedment increased to $15 \%$ and $30 \%$. The DDA cross- 
section of near-field enhancement in Figure 3(c) shows peak intensity shifted toward the AuNSsubstrate interface from $0 \%$ and $30 \%$ embedment; otherwise, the dipole radiation profile changed only slightly. This was consistent with near-constant scattering behavior shown in Figure 3(b). For ITO, a complex-valued lossy dielectric, resonant absorption increased as the AuNS was embedded to $15 \%$ and $30 \%$. The DDA cross-section of near-field enhancement in Figure 3(d) shows peak intensities near the AuNS-ITO interface, larger radius dipole radiation patterns, and higher intensities within the ITO substrate relative to glass, as embedment went from $0 \%$ to $30 \%$. This was consistent with increased (decreased) absorption (scattering) with lossy substrate embedment shown in Figure 3(b). Overall, lossy substrate (i.e., ITO) confined more incident resonant energy than real-valued substrate (i.e., glass).

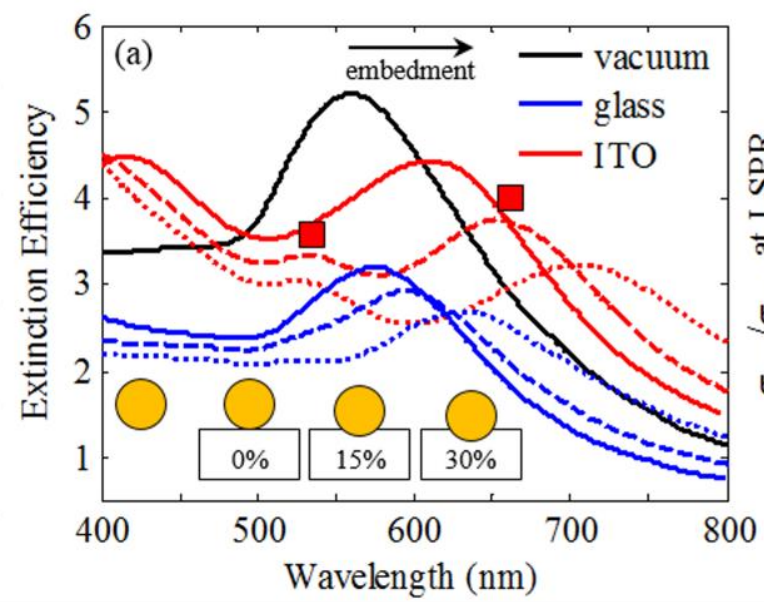

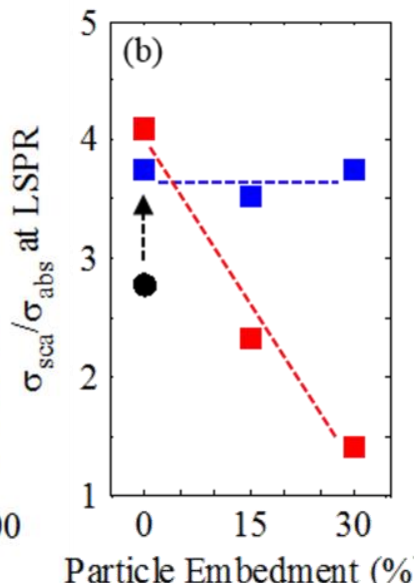
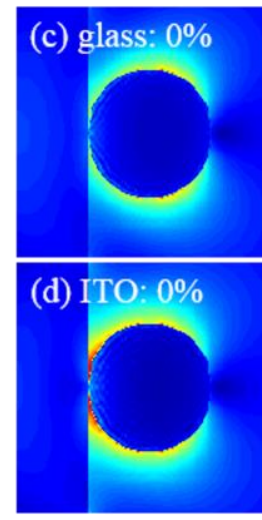

Particle Embedment (\%)

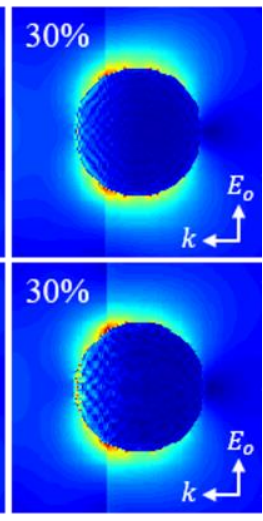

10

Figure 3. Dynamic optical behavior of $80 \mathrm{~nm}$ radius gold nanosphere interacting with complex dielectric substrates. (a) Extinction efficiency spectra for particles embedded 0\% (solid), 15\% (dashed), and 30\% (dotted) of surface area into glass (blue) and ITO (red). Quadrupole (LHS red square) and dipole (RHS red square) LSPR features at $532 \mathrm{~nm}$ and $654 \mathrm{~nm}$ for $15 \%$ ITO embedment were comparable to features in experimental spectra from Figure 1. (b) Ratio of scattering to absorption cross section $\left(\sigma_{s c a} / \sigma_{a b s}\right)$ at resonance for $0 \%, 15 \%$, and $30 \%$ embedded spheres into glass (blue) and ITO (red) relative to vacuum conditions (black). (c) and (d) Electric near-field enhancements $\left(E / E_{o}\right)$ at resonance for $0 \%$ and $30 \%$ embedment in glass and ITO, respectively.

\subsection{Substrate effects on lattices of spheres: coupled lattice resonances and far-field interactions} Polarizability calculated by DDA for a single $80 \mathrm{~nm}$ AuNS (see Figure 2) in a vacuum was inserted into rsa-CDA to calculate far-field extinction spectra for an infinite square array of $80 \mathrm{~nm}$ AuNS, truncated at 90,601 particles for the summation. Figure 4 compares extinction efficiencies calculated with rsa-CDA using Mie (solid) and DDA-calculated (dashed) polarizabilities, respectively. Coupled lattice resonance (CLR) and plasmon resonance [7,57] was within $1 \%$ of those calculated using Mie polarizability. Square lattices of spheres comprising a metasurface support Fano-type coupling between far-field diffraction and near-field LSPR, resulting in a CLR when the lattice constant exceeds the plasmon wavelength [7,12,57]. Diffracted phase interference [38] patterns that coincide with CLRs at $650 \mathrm{~nm}$ and $646 \mathrm{~nm}$ were also observed. Completely destructive interferences at $446 \mathrm{~nm}$ and $631 \mathrm{~nm}$ accrue from diffractive scattering in a $630 \mathrm{~nm}$ square grating. Figure 4 inset expands the spectra from 620 to $680 \mathrm{~nm}$ to show consistency between constructively interfering diffractive phase overlaps calculated with each polarizability. 
The aggregate dipolar lattice plasmon resonance (right-most black circle in main figure) is blueshifted from the single particle LSPR wavelength, consistent with previous results [21,40]. Close correspondence between analytic Mie and DDA-calculated polarizability spectra inserted into the rsa-CDA support multi-scale DDA/rsa-CDA application. Similar correspondence between unabridged DDA results and multi-scale DDA/rsa-CDA simulations has been reported for lattices of nanorings [56].

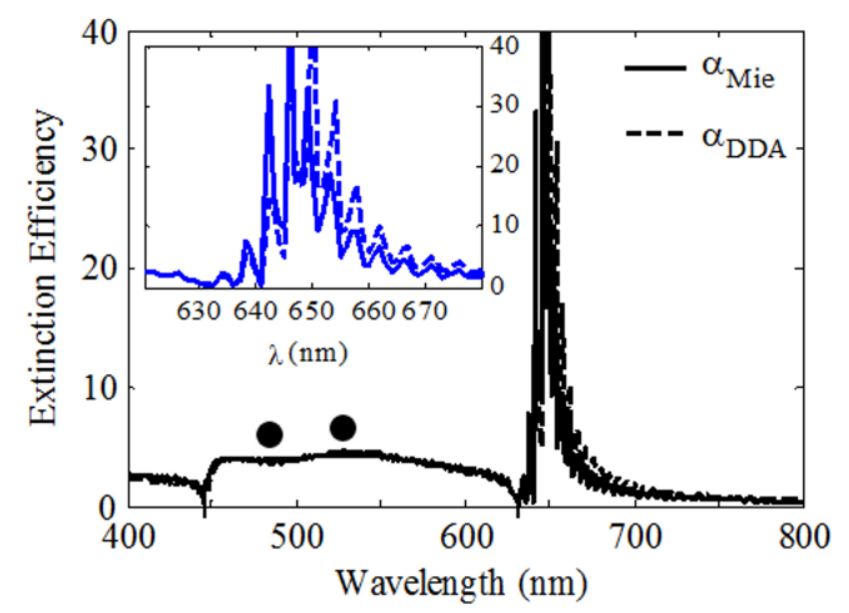

Figure 4. Extinction efficiency spectra for a square lattice of $80 \mathrm{~nm}$ radius Au nanospheres with a lattice constant of $630 \mathrm{~nm}$ in a vacuum evaluated using the rsa-CDA with DDA-extracted polarizability (dashed black) and Mie polarizability (solid black). Diffractive interference points at $446 \mathrm{~nm}$ and $631 \mathrm{~nm}$ arise from the lattice geometry (black cross hatches on X-axis). Inset enlarges phase overlaps in CLR between 620 and $680 \mathrm{~nm}$. Y-axes are truncated on each plot at 40 to increase resolution. Coupled features between $490 \mathrm{~nm}$ and $532 \mathrm{~nm}$ (black circles) were comparable to features in Figure 1 experimental spectra.

Relative to AuNS lattices in homogeneous environments, complex-valued dielectric substrates introduce spectral aberrations due to decoupling of the Fano resonance [22], phase [38] and waveguide [58] effects, new plasmon decay paths [56], and out-of-plane dipole interactions [59]. Non-vacuum environments red-shift the LSPR and lattice constant at unequal rates due to molecular polarization and wavelength contraction, respectively. This decouples the Fano resonance relative to vacuum conditions [22]. A fluid overlayer whose refractive index (RI) matches the substrate can eliminate dielectric discontinuity from influencing extinction spectra [6,12,36,39,60-62]. RI-matched conditions are widely modeled using effective media approximations, but such approximations are limited to real dielectrics with near-zero-dispersion within the spectral region of interest. Instantiations like photovoltaics [63] often preclude implementation or modeling using RI-matching.

Multi-scale DDA/rsa-CDA calculations showed supporting AuNS lattices with glass yielded lossy dipole CLRs relative to the dipole and weak quadrupole CLRs exhibited by ITO. Spectra for 0\% embedment in ITO were closest to vacuum results. Figure 5(a) shows extinction efficiencies from $80 \mathrm{~nm}$ radius AuNS in a square, $630 \mathrm{~nm}$ constant lattice. The CLR wavelength from AuNS atop glass (blue solid) redshifted $31 \mathrm{~nm}$ when embedded 15\% (blue dashed) and 64 $\mathrm{nm}$ when embedded 30\% (blue dotted), relative to $0 \%$ condition. Embedment resulted in substrate 
polarization that red-shifted the LSPR closer to the lattice coupling critical point [7] of $630 \mathrm{~nm}$ (i.e., lattice constant), which resulted in different constructive/destructive interaction between LSPR and lattice diffraction relative to vacuum conditions. This effectively red-shifted coupled resonance behavior (see Figure 4 to compare with vacuum).

Concurrent with these redshifts, CLR bandwidth expanded $83 \mathrm{~nm}$ with $4 \%$ increased intensity for 15\% embedment; and the CLR bandwidth expanded $98 \mathrm{~nm}$ with $39 \%$ increased intensity for $30 \%$ embedment. Bandwidth expands with availability of plasmon decay routes, which arise from radiative scattering for plasmons interacting with a real-valued dielectric [22,64]. Embedment beyond 30\% would decouple the Fano interaction as the single-particle LSPR would red-shift past the $630 \mathrm{~nm}$ lattice constant. Inset to Figure 5(a) shows single-particle LSPR wavelengths (blue squares) redshift as percent embedment increases up to the $630 \mathrm{~nm}$ lattice constant (shown by horizontal black dotted line). The lattice constant must exceed the LSPR to permit Fano coupling. Overall spectral magnitudes in Figure 4 exceed those in Figure 5 due to the smaller cross sectional area of an isolated AuNS relative to a AuNS-substrate target.

Cutting off the portion of the AuNS that had been embedded (15\% or 30\%) red-shifted the LSPR beyond the lattice constant, thus precluding Fano-type coupling. Figure 1(b) shows a cutoff sphere, in contrast to an embedded sphere in Figure 3(a). Fabricated lattices of AuNS appeared to be more cutoff than embedded in SEM analysis, consistent with previous observations [52]. Figure 5(a) shows extinction spectra from lattices of cut-off AuNS (bright green) with 15\% (dashed) and 30\% (dotted) surface area removed. The inset indicates AuNS with greater than $\sim 10 \%$ surface area cut-off do not support Fano resonant dipole coupling with a $630 \mathrm{~nm}$ constant lattice. This is a consequence of the LSPR red-shifting $83 \mathrm{~nm}$ or more due to the disc-shaped surface in contact with the substrate. The major spectral features (bright green circles) were plasmon resonances red-shifted by Fano interference [7]. A minor feature that appeared near 640 $\mathrm{nm}$ for $30 \%$ cut-off AuNS was attributed to weak coupling with higher energy modes (e.g., quadrupole). Minimas that appeared between $575 \mathrm{~nm}-605 \mathrm{~nm}$ that appeared in each AuNS-glass spectra remain to be attributed.

Lossy ITO substrates redshifted the dipole LSPR beyond the $630 \mathrm{~nm}$ order in square lattices of $80 \mathrm{~nm}$ AuNS, precluding dipolar CLRs. Figure 5(b) shows extinction efficiency spectra for arrays of AuNS at 0\% (solid), 15\% (dashed), and 30\% (dotted) surface area embedment (red) or cut-off (dark green) on ITO substrate. A dipole CLR appeared only for 0\%, since the LSPR at $610 \mathrm{~nm}$ was lower than the $630 \mathrm{~nm}$ lattice constant (see Figure 5(b) inset). However, at 15\% and $30 \%$, interaction with the substrate from embedment (red) or cut-off (dark green) produced weak Fano coupling between photon diffraction and (a) quadrupole plasmon [65] and/or (b) ITO scattering (data not shown). Maximas that appeared beyond $700 \mathrm{~nm}$ were dipole plasmon peaks red-shifted by Fano interference [7]. Minimas between $490 \mathrm{~nm}$ and $525 \mathrm{~nm}$ that appeared in each AuNS-ITO spectra remain to be attributed. 

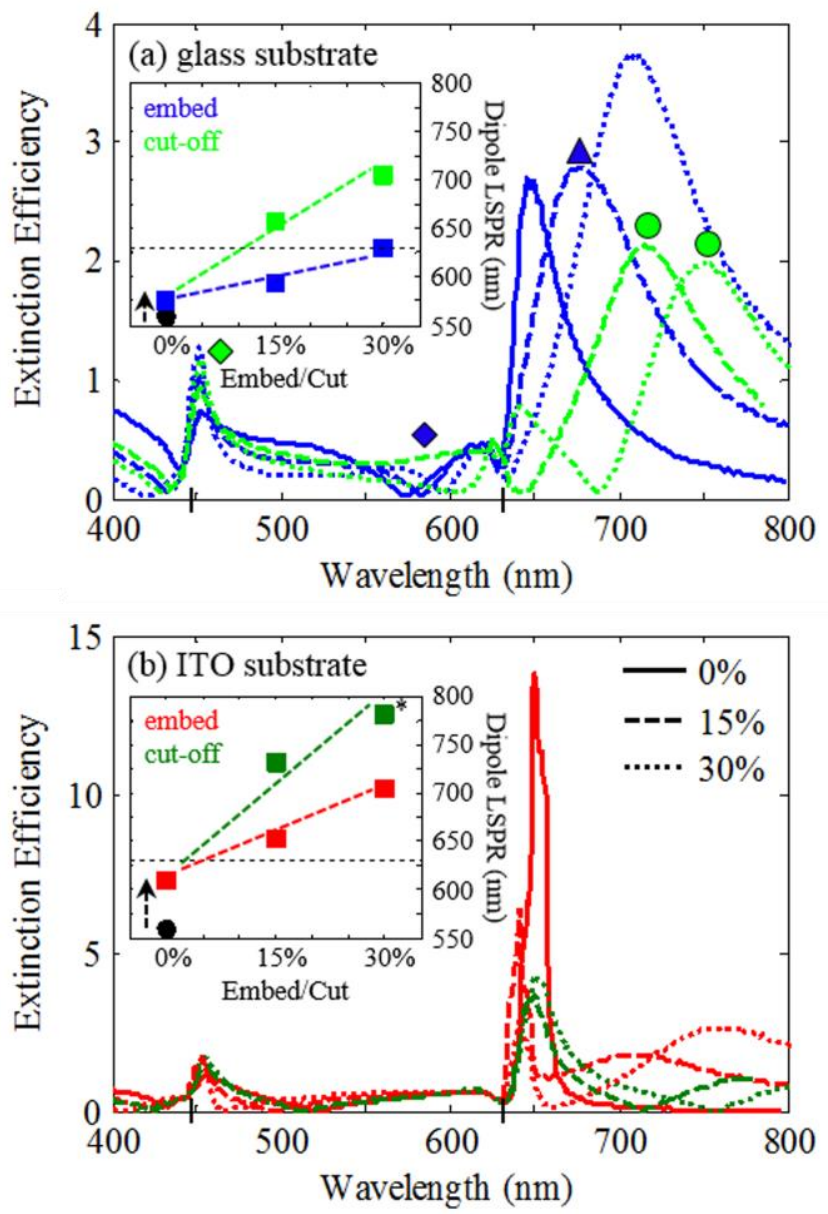

Figure 5. Extinction efficiency spectra for a square lattice $(630 \mathrm{~nm}$ constant) of $80 \mathrm{~nm}$ radius gold nanospheres supported by (a) glass and (b) ITO substrates. Spectra were 10pt Savitzky-Golay smoothed. Spheres were perfectly atop a glass/ITO substrate (blue/red solid line); embedded 15\% (blue/red dashed line); embedded 30\% (blue/red dotted lines); cut off 15\% (bright/dark green dashed lines); or cut off 30\% (bright/dark green dotted lines). Diffractive interference points at $446 \mathrm{~nm}$ and $631 \mathrm{~nm}$ from the lattice geometry are marked (cross hatch on $\mathrm{x}$-axes). Insets show increases in single-particle dipole LSPR wavelength with rise in percent surface area embedded (blue/red) or cut off (bright/dark green). Single-particle dipole LSPR wavelength in vacuum (black circle) and $630 \mathrm{~nm}$ lattice constant (dotted line) are shown for reference. Lattice features in (a) comparable to Figure 1 experimental spectra were the near-580 $\mathrm{nm}$ minima (diamond) and $677 \mathrm{~nm}$ coupled lattice resonance (triangle) of the $15 \%$ glass embed scenario, and lattice-shifted plasmons of the 15\% (716 nm) and 30\% (750 nm) glass cut-off scenarios (circles). Single-particle feature in (b) inset comparable to Figure 1 experimental spectra was the $780 \mathrm{~nm}$ dipole LSPR of the $30 \%$ ITO cut-off scenario.

\subsection{Substrate effects in fabricated square lattices of gold nanospheres}

Spectral features arising from substrate effects on electric resonance modes supported by single and arrayed nanoantennas simulated by multi-scale DDA/rsa-CDA corresponded to previously unattributed spectral extinction features of the fabricated metasurface shown in Figure 1. Features at $528 \mathrm{~nm}$ and $665 \mathrm{~nm}$ had previously been assigned to coupled plasmon and lattice resonance modes using rsa-CDA [21]. But their bandwidth, as well as appearance of other spectral features, were not reproduced by the rsa-CDA using dipole/extended-dipole analytic polarizabilities and effective medium approximations [17,21]. Multi-scale DDA/rsa-CDA simulations in this work 
yielded several resonant features due to substrate interactions that were consistent with experiment: quadrupole and dipole LSPR arising from 15\% embedment of a single AuNS in ITO (Figure 3(a) red squares); coupled plasmon features (Figure 4 black circles); diffractive interference (Figure 5(a) blue diamond) and CLR (Figure 5(a) blue triangle) due to $15 \%$ embedment of arrayed AuNS on glass; lattice-shifted dipole plasmon resonance due to $15 \%$ and $30 \%$ cut-off of arrayed AuNS on glass (Figure 5(a) green circles); and dipole LSPR due to 30\% cut-off of arrayed AuNS on ITO (Figure 5(a) inset square with asterisk). Addition of these simulated features reconstituted the location, bandwidth, and relative magnitudes of corresponding features in the experimental spectra.

Overall, glass appeared to induce far-field diffraction coupled features from lattice arrangement of AuNS while ITO appeared responsible for near-field plasmon features from single AuNS. Figure 6 shows experimental extinction spectra (bottom) on which simulated features are superimposed from glass-induced lattice spectra (LHS charts from spectra in Figure 5(a)) and from ITO-induced particle spectra (RHS charts from Figures 3(a) and 5(b)). Symbols and arrows connect features from underlying spectra to components of the measured spectra. Resonant signals of five AuNS-substrate pairs were observed in the experimental spectra instead of a singular scenario. This was consistent with sample irregularities: appearance of disordered AuNS in the spectrometer field of view in addition to the lithographed lattice; colloidal gold deposition during resist liftoff; and subwavelength ITO thickness which permitted underlying glass to interact with diffractive modes.

From the LHS of the bottom chart in Figure 6, the $\sim 450 \mathrm{~nm}$ shoulder (bright green diamond) corresponds to a constructive interference present throughout Figures 5(a) and 5(b). It is shown represented by arrayed, 15\% cut-off AuNS on glass. Coupled plasmon valley and resonance at $490 \mathrm{~nm}$ and $528 \mathrm{~nm}$, respectively (black circles), were anticipated by CDA models of AuNS lattices in vacuum (Figure 4). The $528 \mathrm{~nm}$ resonance also corresponded to the location of a quadrupole feature (red square) from a single AuNS embedded 15\% in ITO. The local minima at $580 \mathrm{~nm}$ (blue diamond) appeared characteristic of AuNS-glass interactions; arrayed AuNS embedded $15 \%$ in glass is illustrated. It is also noted that a minima near $580 \mathrm{~nm}$ was observed from single AuNS embedded $15 \%$ in ITO.

The major measured feature at $665 \mathrm{~nm}$ corresponded to a simulated CLR from arrayed AuNS embedded $15 \%$ in glass (blue triangle) with an underlying LSPR characteristic of single AuNS embedded 15\% in ITO (red square). The $700 \mathrm{~nm}$ shoulder appeared due to a coupled lattice plasmon from arrayed AuNS cutoff $15 \%$ on glass (green circle). Its refractive index sensitivity, shown via water immersion in Figure 1, supports attribution as a coupled feature. A feature appeared near $650 \mathrm{~nm}$ in the measured spectra when the sample was immersed in water, suggesting an LSPR mode underlay the major CLR feature. LSPR of isolated AuNS embedded 15\% in ITO was the most probable origin of this underlying feature. Weak coupling between arrayed AuNS embedded 15\% in ITO may have supplemented the $650 \mathrm{~nm}$ and $700 \mathrm{~nm}$ shoulder features, but is not shown for brevity. Overall bandwidth of the $665 \mathrm{~nm}$ major feature could be due in part to 
LSPR from singled AuNS cut-off 15\% on glass (not shown in Figure 6). Unordered AuNS were observed via SEM in the periphery of area from which spectra was acquired.

Local maxima observed at $750 \mathrm{~nm}$ and $785 \mathrm{~nm}$ corresponded to a lattice-shifted plasmon mode (bright green circle) from arrayed AuNS cut-off $30 \%$ on glass, and a LSPR mode (dark green square) from a single AuNS cut-off $30 \%$ on ITO, respectively. The local maxima at $750 \mathrm{~nm}$ did not broaden upon experimental submersion in water and its RI sensitivity was characteristic of a plasmon, consistent with the former attribution. The local maxima at $785 \mathrm{~nm}$ broadened when submerged in water, as expected from an LSPR from single AuNS. Other similarities existed between experimental and simulated spectra that were less prevalent than those shown.

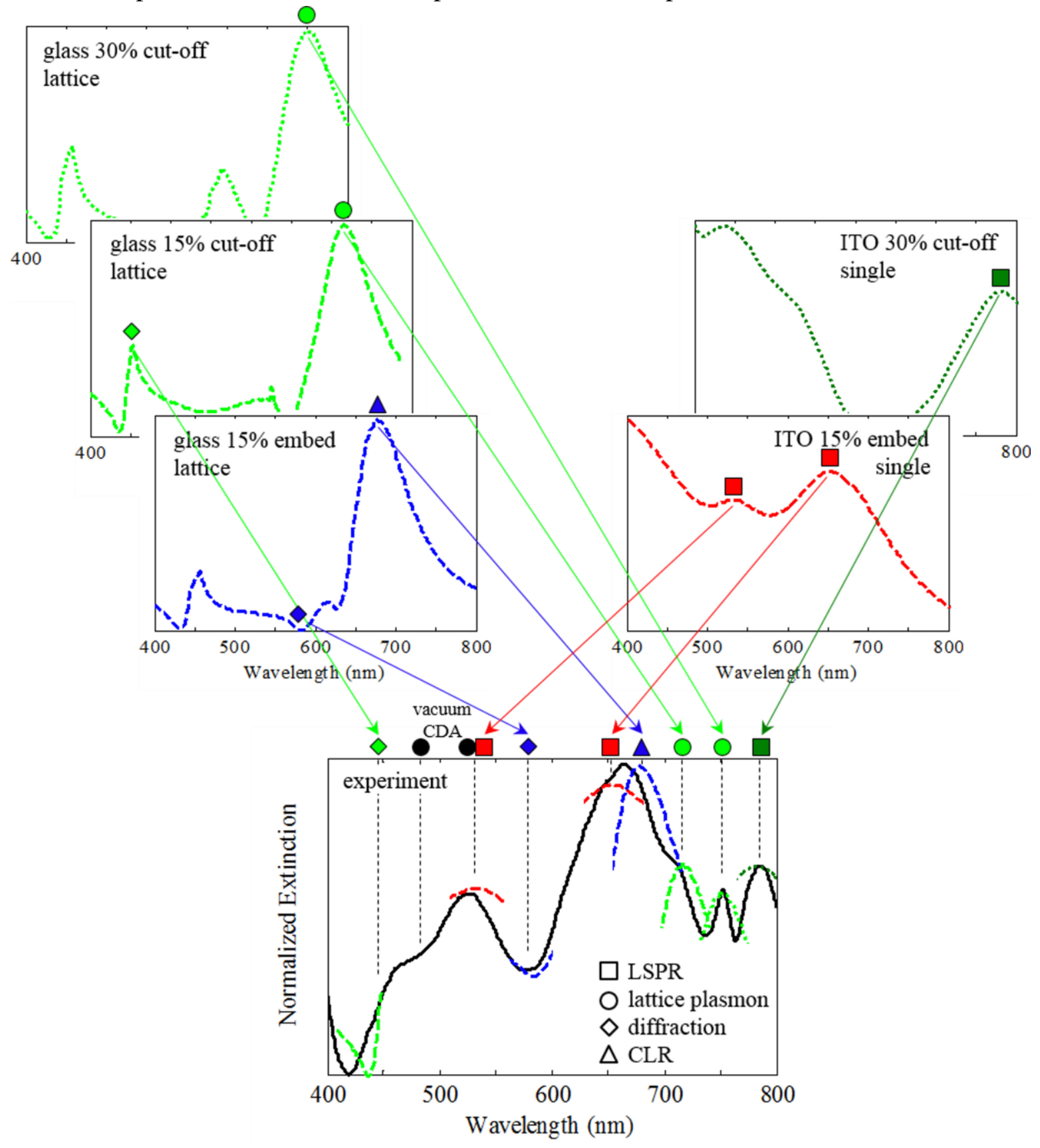


Figure 6. Experimental spectra from Figure 1 (black line) with corresponding features from simulated single-particle modes (RHS) due to LSPR (squares) and lattice modes (LHS) due to plasmons (circles), coupled resonances (triangles), and diffractive interference (diamonds). Simulations are fully described in Figures 3 and 5.

To summarize, multi-scale DDA/rsa-CDA simulations showing effects of substrate interactions on far-field spectra of single and arrayed AuNS embedded or cut-off on glass and ITO provided possible sources for features observed in experimental spectra. These features were not attributable by previous simulations of AuNS that used dipole or extended-dipole polarizabilities and mass-averaged, dispersion-less dielectric values or analytical Maxwell-Garnett effective medium approaches. Simulated modes that corresponded to observed features included LSPR (square), lattice-shifted plasmon (circle), diffraction (diamond), and CLR (triangle). Simulations were based on physical features observed by SEM analysis; e.g., AuNS appeared cut-off [52]. Attributions were assigned based on changes in feature location and bandwidth after immersion in water, e.g., LSPR peaks red-shift less and broaden more relative to coupled resonances in response to a change in local dielectric [22]. Neither location nor bandwidth of simulated features were altered to correlate with experimental data. Location and bandwidth of spectral features intrinsically represent electric resonance modal induction and losses. Simulated features were vertically offset in some cases when overlaid on experimental data. Such offsets can arise in practice due to the quantity of sampled nanoparticles, spectrometer setup (e.g., focal length and probe size), quality of metallic film lift off [52], lattice constant disorder [61], and morphological antenna defects.

\section{Conclusion}

Effects of real-valued (e.g., glass) and lossy, complex (e.g., ITO) dielectrics on plasmon activity of noble metal nanosphere lattices were simulated using DDA to examine near-field LSPR of single AuNS, and by integrating DDA-calculated polarizabilities into rsa-CDA to examine farfield lattice interactions. The real-valued dielectric red-shifted and broadened the LSPR via emergence of additional radiative loss modes. The complex-valued dielectric further redshifted the LSPR and increased resonant absorption. Inserting substrate-altered polarizabilities calculated by DDA for nanoantenna at $100 \mathrm{~nm}$ scales into rsa-CDA computation at $>100,000 \mathrm{~nm}$ scales for nanoantenna lattices elucidated dependence of diffractive spectral features (e.g., coupled lattice resonance) on glass substrates due to increased resonant scattering, while dipole plasmon dependence on ITO due to enhanced absorption and energy confinement. Embedding or cutting off the AuNS typically red-shifted the LSPR beyond the Fano coupling regime (i.e., $630 \mathrm{~nm}$ constant exceeding LSPR). This precluded CLR emergence and resulted in red-shifted plasmon modes from Fano interference and weakly coupled quadrupole lattice resonances. The energy, bandwidth, and relative intensity of these modes, which had not been previously described, coincided with experimental spectral features whose origin had been unknown. Multi-scale DDA/rsa-CDA simulations make possible efficient characterization of substrate effects on localized and coupled plasmon resonances in metasurfaces for optoelectronic platforms. 


\section{Acknowledgments}

This work was supported in part by NSF CBET-1134222, NSF ECCS-1006927, NSF EEC1260301, NSF Graduate Research Fellowship awarded to G.T.F., ASSET Initiative Undergraduate Research Internship awarded to M.S., the University of Arkansas Foundation, and the Walton Family Charitable Foundation. DDA simulations were performed on the supercomputers of the Arkansas High Performance Computing Center, supported by NSF-918970 and NSF-959124. Any opinions, findings, and conclusions or recommendations expressed in this material are those of the authors and do not necessarily reflect the views of the National Science Foundation. G.T.F. performed numerical modeling and prepared text and figures for the manuscript. P.B. fabricated the sample and performed characterization. M.S. performed numerical modeling. D.D. aided in numerical modeling and initially evaluated the modeling technique. D.K.R. directed the work and refined compilation of figures and text. 


\section{References}

[1] Mayer KM, Hafner JH. Localized surface plasmon resonance sensors. Chem Rev 2011;111:3828-57. doi:10.1021/cr100313v.

[2] Mossotti OF. Mem Di Methem E Fis Modena 1850;24:49.

[3] Clausius R. L. Die Mech U'grmetheorie 1879;2:62.

[4] Wang ZL, Wu W. Nanotechnology-enabled energy harvesting for self-powered micro/nanosystems. Angew Chemie 2012;51:11700-21. doi:10.1002/anie.201201656.

[5] Brongersma ML, Halas NJ, Nordlander P. Plasmon-induced hot carrier science and technology. Nat Nanotechnol 2015;10:25-34. doi:10.1038/nnano.2014.311.

[6] Offermans P, Schaafsma MC, Rodriguez SRK, Zhang Y, Crego-Calama M, Brongersma $\mathrm{SH}$, et al. Universal scaling of the figure of merit of plasmonic sensors. ACS Nano 2011;5:5151-7. doi:10.1021/nn201227b.

[7] Forcherio GT, Blake P, DeJarnette D, Roper DK. Nanoring structure, spacing, and local dielectric sensitivity for plasmonic resonances in Fano resonant square lattices. Opt Express 2014;22:17791-804. doi:10.1364/OE.22.017791.

[8] Ozbay E. Plasmonics: merging photonics and electronics at nanoscale dimensions. Science 2006;311:189-93. doi:10.1126/science.1114849.

[9] Brongersma ML, Shalaev VM. The case for plasmonics. Science 2010;328:440-1. doi:10.1126/science.1186905.

[10] Kauranen M, Zayats A V. Nonlinear plasmonics. Nat Photonics 2012;6:737-48. doi:10.1038/nphoton.2012.244.

[11] McMahon MD, Ferrara D, Bowie CT, Lopez R, Haglund Jr. RF. Second harmonic generation from resonantly excited arrays of gold nanoparticles. Appl Phys B 2007;87:259-65. doi:10.1007/s00340-006-2569-3.

[12] Luk'yanchuk B, Zheludev NI, Maier SA, Halas NJ, Nordlander P, Giessen H, et al. The Fano resonance in plasmonic nanostructures and metamaterials. Nat Mater 2010;9:70715. doi:10.1038/nmat2810.

[13] Mak KF, Lee C, Hone J, Shan J, Heinz TF. Atomically thin MoS2: a new direct-gap semiconductor. Phys Rev Lett 2010;105:136805. doi:10.1103/PhysRevLett.105.136805.

[14] Garnett JCM. Colours in metal glasses and in metallic films. Philos Trans R Soc A 1904;203:385-420. doi:10.1098/rsta.1904.0024. 
[15] Niklasson GA, Granqvist CG, Hunderi O. Effective medium models for the optical properties of inhomogeneous materials. Appl Opt 1981;20:26-30.

[16] Toudert J, Simonot L, Camelio S, Babonneau D. Advanced optical effective medium modeling for a single layer of polydisperse ellipsoidal nanoparticles embedded in a homogeneous dielectric medium: Surface plasmon resonances. Phys Rev B 2012;86:045415. doi:10.1103/PhysRevB.86.045415.

[17] Curry A, Nusz G, Chilkoti A, Wax A. Substrate effect on refractive index dependence of plasmon resonance for individual silver nanoparticles observed using darkfield microspectroscopy. Opt Express 2005;13:2668-77.

[18] Pastoriza-Santos I, Gomez D, Perez-Juste J, Liz-Marzan LM, Mulvaney P. Optical properties of metal nanoparticle coated silica spheres: a simple effective medium approach. Phys Chem Chem Phys 2004;6:5056-60. doi:10.1039/b405157b.

[19] Forcherio GT, Roper DK. Optical attenuation of plasmonic nanocomposites within photonic devices. Appl Opt 2013;52:6417-27. doi:10.1364/AO.52.006417.

[20] Roper DK, Ahn W, Taylor B, Asen AGD. Enhanced spectral sensing by electromagnetic coupling with localized surface plasmons on subwavelength structures. IEEE Sensors 2010;10:531-40.

[21] Blake P, Kühne S, Forcherio GT, Roper DK. Diffraction in nanoparticle lattices increases sensitivity of localized surface plasmon resonance to refractive index changes. $\mathrm{J}$ Nanophotonics 2014;8:083084. doi:10.1117/1.JNP.8.083084.

[22] DeJarnette D, Norman J, Roper DK. Attribution of Fano resonant features to plasmonic particle size, lattice constant, and dielectric wavenumber in square nanoparticle lattices. Photonics Res 2014;2:15-23. doi:10.1364/PRJ.2.000015.

[23] Larsson EM, Alegret J, Käll M, Sutherland DS. Sensing characteristics of NIR localized surface plasmon resonances in gold nanorings for application as ultrasensitive biosensors. Nano Lett 2007;7:1256-63. doi:10.1021/n10701612.

[24] Dmitriev A, Hägglund C, Chen S, Fredriksson H, Pakizeh T, Käll M, et al. Enhanced nanoplasmonic optical sensors with reduced substrate effect. Nano Lett 2008;8:3893-8. doi:10.1021/n18023142.

[25] Vernon KC, Funston AM, Novo C, Gómez DE, Mulvaney P, Davis TJ. Influence of particle-substrate interaction on localized plasmon resonances. Nano Lett 2010;10:2080 6. doi:10.1021/nl100423z.

[26] Knight MW, Wu Y, Lassiter JB, Nordlander P, Halas NJ. Substrates matter: influence of an adjacent dielectric on an individual plasmonic nanoparticle. Nano Lett 2009;9:218892. doi:10.1021/n1900945q. 
[27] Malinsky MD, Kelly KL, Schatz GC, Van Duyne RP. Nanosphere lithography: effect of substrate on the localized surface plasmon resonance spectrum of silver nanoparticles. J Phys Chem B 2001;105:2343-50. doi:10.1021/jp002906x.

[28] Ringe E, McMahon JM, Sohn K, Cobley C, Xia Y, Huang J, et al. Unraveling the effects of size, composition, and substrate on the localized surface plasmon resonance frequencies of gold and silver nanocubes: A systematic single-particle approach. J Phys Chem C 2010;114:12511-6. doi:10.1021/jp104366r.

[29] Mahmoud MA, Chamanzar M, Adibi A, El-Sayed M A. Effect of the dielectric constant of the surrounding medium and the substrate on the surface plasmon resonance spectrum and sensitivity factors of highly symmetric systems: silver nanocubes. J Am Chem Soc 2012;134:6434-42. doi:10.1021/ja300901e.

[30] Hawes EA, Hastings JT, Crofcheck C, Mengüç MP. Spectrally selective heating of nanosized particles by surface plasmon resonance. J Quant Spectrosc Radiat Transf 2007;104:199-207. doi:10.1016/j.jqsrt.2006.07.020.

[31] Chou A, Vernon KC, Piro L, Radi B, Jaatinen EA, Davis TJ. Predicting the localized surface plasmon resonances of spherical nanoparticles on a substrate: electrostatic eigenmode method. J Phys Chem C 2012;116:26517-22. doi:10.1021/jp307510e.

[32] García de Abajo FJ. Optical excitations in electron microscopy. Rev Mod Phys 2010;82:209-75. doi:10.1103/RevModPhys.82.209.

[33] Yurkin MA, Hoekstra AG. The discrete dipole approximation: An overview and recent developments. J Quant Spectrosc Radiat Transf 2007;106:558-89. doi:10.1016/j.jqsrt.2007.01.034.

[34] Penttilä A, Zubko E, Lumme K, Muinonen K, Yurkin MA, Draine B, et al. Comparison between discrete dipole implementations and exact techniques. J Quant Spectrosc Radiat Transf 2007;106:417-36. doi:10.1016/j.jqsrt.2007.01.026.

[35] Kelly KL, Coronado E, Zhao LL, Schatz GC. The optical properties of metal nanoparticles: the influence of size, shape, and dielectric environment. J Phys Chem B 2003;107:668-77. doi:10.1021/jp026731y.

[36] Hicks EM, Zou S, Schatz GC, Spears KG, Van Duyne RP, Gunnarsson L, et al. Controlling plasmon line shapes through diffractive coupling in linear arrays of cylindrical nanoparticles fabricated by electron beam lithography. Nano Lett 2005;5:1065-70. doi:10.1021/n10505492.

[37] Jain PK, Eustis S, El-Sayed MA. Plasmon coupling in nanorod assemblies: optical absorption, discrete dipole approximation simulation, and exciton-coupling model. J Phys Chem B 2006;110:18243-53. doi:10.1021/jp063879z. 
[38] DeJarnette D, Norman J, Roper DK. Spectral patterns underlying polarization-enhanced diffractive interference are distinguishable by complex trigonometry. Appl Phys Lett 2012;101:183104. doi:10.1063/1.4764943.

[39] Auguié B, Barnes W. Collective Resonances in Gold Nanoparticle Arrays. Phys Rev Lett 2008;101:143902. doi:10.1103/PhysRevLett.101.143902.

[40] DeJarnette D, Roper DK, Harbin B. Geometric effects on far-field coupling between multipoles of nanoparticles in square arrays. J Opt Soc Am B 2012;29:88-100. doi:10.1364/JOSAB.29.000088.

[41] Mary A, Koller D, Hohenau A, Krenn J, Bouhelier A, Dereux A. Optical absorption of torus-shaped metal nanoparticles in the visible range. Phys Rev B 2007;76:245422. doi:10.1103/PhysRevB.76.245422.

[42] DeJarnette D, Blake P, Forcherio GT, Roper DK. Far-field Fano resonance in nanoring arrays modeled from extracted, point dipole polarizability. J Appl Phys 2014;115:024306.

[43] Simsek E. Full analytical model for obtaining surface plasmon resonance modes of metal nanoparticle structures embedded in layered media. Opt Express 2010;18:1722-33. doi:10.1364/OE.18.001722.

[44] Draine BT, Flatau PJ. Discrete-dipole approximation for scattering calculations. J Opt Soc Am A 1994;11:1491-9. doi:10.1364/JOSAA.11.001491.

[45] Draine BT, Flatau PJ. Discrete-dipole approximation for periodic targets: theory and tests. J Opt Soc Am A 2008;25:2693-703.

[46] Flatau PJ, Draine BT. Fast near field calculations in the discrete dipole approximation for rectangular rectilinear grids. Opt Express 2012;20:1247-52.

doi:http://dx.doi.org/10.1364/OE.20.001247.

[47] Seeram M, Forcherio GT, Blake P, Roper DK. Shape Generator for the Discrete Dipole Approximation. nanoHUB 2015. https://nanohub.org/resources/22363.

[48] Gutkowicz-Krusin D, Draine BT. Propagation of Electromagnetic Waves on a Rectangular Lattice of Polarizable Points. Http://arxiv.org/abs/astro-ph/0403082 2004.

[49] Rubin M. Optical properties of soda lime silica glasses. Sol Energy Mater 1985;12:27588. doi:10.1016/0165-1633(85)90052-8.

[50] König TAF, Ledin PA, Kerszulis J, Mahmoud MA, El-Sayed MA, Reynolds JR, et al. Electrically tunable plasmonic behavior of nanocube-polymer nanomaterials induced by a redox-active electrochromic polymer. ACS Nano 2014;8:6182-92.

doi:10.1021/nn501601e. 
[51] Johnson PB, Christy RW. Optical Constants of the Noble Metals. Phys Rev B 1972;6:4370-9. doi:10.1103/PhysRevB.6.4370.

[52] Blake P, Ahn W, Roper DK. Enhanced uniformity in arrays of electroless plated spherical gold nanoparticles using tin presensitization. Langmuir 2010;26:1533-8.

doi:10.1021/la903985m.

[53] Blake P, Obermann J, Harbin B, Roper DK. Enhanced nanoparticle response from coupled dipole excitation for plasmon sensors. IEEE Sensors 2011;11:3332-40.

doi:10.1109/JSEN.2011.2158417.

[54] Aizpurua J, Blanco L, Hanarp P, Sutherland DS, Käll M, Bryant GW, et al. Light scattering in gold nanorings. J Quant Spectrosc Radiat Transf 2004;89:11-6.

doi:10.1016/j.jqsrt.2004.05.007.

[55] An W, Zhu T, Zhu Q. Numerical investigation of radiative properties and surface plasmon resonance of silver nanorod dimers on a substrate. J Quant Spectrosc Radiat Transf 2014;132:28-35. doi:10.1016/j.jqsrt.2013.01.013.

[56] DeJarnette D, Roper DK. Electron energy loss spectroscopy of gold nanoparticles on graphene. J Appl Phys 2014;116:054313. doi:10.1063/1.4892620.

[57] Francescato Y, Giannini V, Maier S A. Plasmonic systems unveiled by Fano resonances. ACS Nano 2012;6:1830-8. doi:10.1021/nn2050533.

[58] Linden S, Kuhl J, Giessen H. Controlling the interaction between light and gold nanoparticles: selective suppression of extinction. Phys Rev Lett 2001;86:4688-91. doi:10.1103/PhysRevLett.86.4688.

[59] Zhou W, Odom TW. Tunable subradiant lattice plasmons by out-of-plane dipolar interactions. Nat Nanotechnol 2011;6:423-7. doi:10.1038/nnano.2011.72.

[60] Auguié B, Bendaña XM, Barnes WL, García De Abajo FJ. Diffractive arrays of gold nanoparticles near an interface: critical role of the substrate. Phys Rev B 2010;82:155447. doi:10.1103/PhysRevB.82.155447.

[61] Auguié B, Barnes WL. Diffractive coupling in gold nanoparticle arrays and the effect of disorder. Opt Lett 2009;34:401-3. doi:10.1364/OL.34.000401.

[62] Wang H, Vial A. Tunability of LSPR using gold nano-particles embedded in a liquid crystal cell. J Quant Spectrosc Radiat Transf 2014;146:492-8.

doi:10.1016/j.jqsrt.2014.02.008.

[63] Diukman I, Orenstein M. How front side plasmonic nanostructures enhance solar cell efficiency. Sol Energy Mater Sol Cells 2011;95:2628-31. doi:10.1016/j.solmat.2011.05.019. 
[64] Jensen T, Kelly KL, Lazarides A, Schatz GC. Electrodynamics of noble metal nanoparticles and nanoparticle clusters. J Clust Sci 1999;10:295-317. doi:10.1023/A:1021977613319.

[65] DeJarnette D, Jang GG, Blake P, Roper DK. Polarization angle affects energy of plasmonic features in Fano resonant regular lattices. J Opt 2014;16:105006. doi:10.1088/2040-8978/16/10/105006. 